\title{
An Efficient Numerical Method in Calculating the Electrical Impedance Different Modes of AT-Cut Quartz Crystal Resonator
}

\author{
S.Y. Pao ${ }^{(1)}$, M.K. Chao ${ }^{(2)}$, C.S. Lam ${ }^{(2)}$, and P.Z. Chang ${ }^{(1)}$ \\ (1) Institute of Applied Mechanics, National Taiwan University, Taipei, Taiwan \\ (2) TXC Corporation, Ping Cheng City, Taoyuan County, Taiwan
}

\begin{abstract}
Lee and Brebbia in $\mathbf{1 9 7 8}$ presented a finite element analysis (FEA) of AT-cut quartz crystal resonator by including the thickness-shear branches in the length-width directions and the flexural branch in the thickness direction based on Mindlin's 2-D elastic equations. The formulation was pure elastic and electrical impedance of different modes could not be calculated since piezoelectric effect and damping were not included. In the years after, many FEA-based studies were conducted using either the full-blown 3-D elastic-piezoelectric model or the simplified Mindlin's 2-D elastic-piezoelectric model. Such formulations are quite complicated and numerically time-consuming in computation. Furthermore, one experienced tremendous difficulty in resolving the many eigenmodes in the 3-D analysis.
\end{abstract}

In this paper, we first solve for the eigenmodes of the AT-cut quartz crystal resonator without piezoelectric effect using LeeBrebbia's FEA method based on Mindlin's 2-D elastic equations. By assuming weak piezoelectric coupling, we rearrange the electrical potential to the right hand side of the elasticpiezoelectric equations of motion. These terms become the forcing terms of the pure elastic equations of motion. We also consider the constant damping terms in the equations of motion. Then we use the mode superposition method to calculate the weight of each eigenmode. The elastic displacement field with electrical potential effect can then be calculated as the weighting sum of each eigenmode. The electrical charges on the quartz crystal resonator can be obtained from the elastic displacement and electrical potential field at each node point. Finally, we can calculate the electrical impedance from the electrical charges and the potential. This method is considered much more efficient than the full-blown 3-D elastic-piezoelectric analysis or the simplified Mindlin's 2-D elastic-piezoelectric analysis.

Keywords- AT-cut quartz resonator; electrrical impedance; finite element analysis; mode superposition method

\section{INTRODUCTION}

Because of its high Q and high temperature stability, ATcut quartz is widely use in oscillators as the heart of the circuit. Along with the personal mobile telecommunication process, the miniaturization of circuit components, including resonators and oscillators, is inevitable. As the size of quartz resonators becomes smaller and smaller, the bonding, beveling and electrode effects are much more important than that in the bigger size device in the past. The mode coupling and temperature stability problems are also more complex in smaller package size. If an engineer still only uses some simple experience formulae or trial and error to design resonators, it is time-consuming and therefore expensive. And it is more difficult to induce empirical formulae by trial and error. Because of the demand of more precise design tool, some commercial finite element analysis (FEA) software can be used to simulate piezoelectric resonators. But AT-cut quartz resonators are strip or round plates and the diameter (or length) to thickness ratio is typical 50 to 100 , so it is very hard to take both thickness direction resolution and element aspect ratio into consideration as meshing the quartz plate. However, how to build the model for the electrode and beveling chip is also a challenge in commercial FEA tool. Besides precision, efficiency is also very important for a design engineer. If it spends tens hours to several days to simulate only one case, the design cycle will be too long and impractical. Because of these reasons, we develop an efficient numerical method and use FORTRAN program to calculate the electrical impedance different modes of AT-cut quartz crystal resonator. This method can simulate not only pure mechanical vibration behavior but also electrical impedance response, and is both precise enough and efficient for design engineers.

\section{THEORY OF PIEZOELECTRIC FINITE ELEMENT}

According to Mindlin's two-dimensional plate equations [1] and neglecting the coupling with extensional motions by setting $\bar{c}_{14}=\bar{c}_{34}=c_{56}=0$, we obtain:

$$
\begin{aligned}
& Q_{1,1}+Q_{3,3}=2 b \rho \ddot{u}_{2} \\
& \mathrm{M}_{1,1}+\mathrm{M}_{5,3}-Q_{1}=\frac{2}{3} b^{3} \rho \ddot{\psi}_{1} \\
& \mathrm{M}_{5,1}+\mathrm{M}_{3,3}-Q_{3}=\frac{2}{3} b^{3} \rho \ddot{\psi}_{3}
\end{aligned}
$$

where $\mathrm{M}_{1}$ and $\mathrm{M}_{3}$ are bending moment; $\mathrm{M}_{5}$ is twist moment; Q is shear force; $\mathrm{b}$ is half thickness; $\rho$ is density of quartz; $\psi_{1}$ and $\psi_{3}$ are the rotating angle along axis $x_{3}$ and $x_{1}$.

For strip quartz plate, we use the nine-node quadratic element as the FEA elements. 

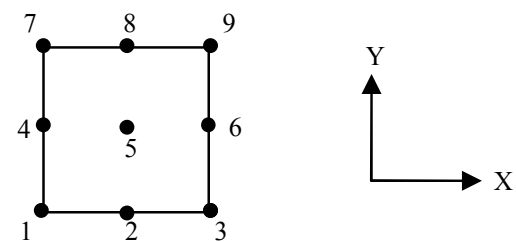

Figure 1. Nine-node quadratic element

Then for an element with dimension $\mathrm{W}$ and $\mathrm{L}$ along $\mathrm{x}$ and $\mathrm{y}$ directions, respectively, the interpolation functions are [2]:

$$
\varphi=\left[\begin{array}{lll}
\varphi_{1} & \varphi_{4} & \varphi_{7} \\
\varphi_{2} & \varphi_{5} & \varphi_{8} \\
\varphi_{3} & \varphi_{6} & \varphi_{9}
\end{array}\right]
$$

where

$$
\begin{aligned}
& \varphi_{1}=\left(1-\frac{2 x}{W}\right)\left(1-\frac{x}{W}\right)\left(1-\frac{2 y}{L}\right)\left(1-\frac{y}{L}\right) \\
& \varphi_{2}=\frac{4 x}{W}\left(1-\frac{x}{W}\right)\left(1-\frac{2 y}{L}\right)\left(1-\frac{y}{L}\right) \\
& \varphi_{3}=\frac{x}{W}\left(\frac{2 x}{W}-1\right)\left(1-\frac{x}{W}\right)\left(1-\frac{2 y}{L}\right)\left(1-\frac{y}{L}\right) \\
& \varphi_{4}=\left(1-\frac{2 x}{W}\right)\left(1-\frac{x}{W}\right) \frac{4 y}{L}\left(1-\frac{y}{L}\right) \quad \varphi_{5}=\frac{4 x}{W}\left(1-\frac{x}{W}\right) \frac{4 y}{L}\left(1-\frac{y}{L}\right) \\
& \varphi_{6}=\frac{x}{W}\left(\frac{2 x}{W}-1\right) \frac{4 y}{L}\left(1-\frac{y}{L}\right) \quad \varphi_{7}=\left(1-\frac{2 x}{W}\right)\left(1-\frac{x}{W}\right) \frac{y}{L}\left(\frac{2 y}{L}-1\right) \\
& \varphi_{8}=\frac{4 x}{W}\left(1-\frac{x}{W}\right) \frac{y}{L}\left(\frac{2 y}{L}-1\right) \quad \varphi_{9}=\frac{x}{W}\left(\frac{2 x}{W}-1\right) \frac{y}{L}\left(\frac{2 y}{L}-1\right)
\end{aligned}
$$

Based on the Mindlin's 2D model, there are three degrees of freedom for each node in the element (one displacement and two rotating angle), so the interpolation function of one element is:

$$
\phi=\left[\begin{array}{l}
\phi_{1} \\
\phi_{2} \\
\phi_{3}
\end{array}\right]_{3 \times 27}
$$

where

$$
\begin{aligned}
& \phi_{1}=\left[\begin{array}{lllllllllllll}
\varphi_{1} & 0 & 0 & \varphi_{2} & 0 & 0 & \varphi_{3} & 0 & 0 & \cdots & \varphi_{9} & 0 & 0
\end{array}\right]_{1 \times 27} \\
& \phi_{2}=\left[\begin{array}{lllllllllllll}
0 & \varphi_{1} & 0 & 0 & \varphi_{2} & 0 & 0 & \varphi_{3} & 0 & \cdots & 0 & \varphi_{9} & 0
\end{array}\right]_{1 \times 27}(3 \mathrm{a}) \\
& \phi_{2}=\left[\begin{array}{lllllllllllll}
0 & 0 & \varphi_{1} & 0 & 0 & \varphi_{2} & 0 & 0 & \varphi_{3} & \cdots & 0 & 0 & \varphi_{9}
\end{array}\right]_{1 \times 27}
\end{aligned}
$$

By the finite element displacement method for Mindlin's 2D model developed by P.C.Y. Lee et al. [3], the displacementnodal displacement relationship is:

$$
\{U\}=\left\{\begin{array}{l}
u_{2} \\
\psi_{1} \\
\psi_{3}
\end{array}\right\}
$$

The displacement vector at $\mathrm{i}^{\text {th }}$ node of an element is:

$$
u^{i}=\left\{\begin{array}{l}
u_{2}^{i} \\
\psi_{1}^{i} \\
\psi_{3}^{i}
\end{array}\right\}
$$

So, the element nodal displacement $\mathrm{U}^{\mathrm{e}}$ is:

$$
\left\{U^{e}\right\}=\left\{\begin{array}{c}
u^{1} \\
u^{2} \\
\vdots \\
u^{9}
\end{array}\right\}_{27 \times 1}
$$

Then, the displacement field, $\mathrm{U}$, within the element is

$$
U=[\phi]\left\{U^{e}\right\}
$$

The equation of motion for an element (pure mechanical vibration, without damping):

$$
\left[M^{e}\right]\left\{\ddot{U}^{e}\right\}+\left[K^{e}\right]\left\{U^{e}\right\}=\{0\}
$$

where

$$
\begin{aligned}
& {\left[M^{e}\right]=\int_{A}[\phi]^{T}[m][\phi] d A} \\
& {[m]=\left[\begin{array}{ccc}
2 b \rho(1+R) & 0 & 0 \\
0 & \frac{2}{3} b^{3} \rho(1+3 R) & 0 \\
0 & 0 & \frac{2}{3} b^{3} \rho(1+3 R)
\end{array}\right]}
\end{aligned}
$$

$R=2 \rho^{\prime} b^{\prime} / \rho b$ is the ratio of the mass of electrodes to the mass of the plate per unit area (if no electrodes, $R=0$ ) and

$$
\begin{aligned}
& {\left[K^{e}\right]=\int_{A}\left(\left[B_{b}\right]^{T}\left[D_{b}\right]\left[B_{b}\right]+\left[B_{t}\right]^{T}\left[D_{t}\right]\left[B_{t}\right]\right) d A} \\
& {\left[B_{b}\right]=\left[\begin{array}{c}
\phi_{2,1} \\
\phi_{3,3} \\
\phi_{3,1}+\phi_{2,3}
\end{array}\right]_{3 \times 27}} \\
& {\left[B_{t}\right]=\left[\begin{array}{c}
\phi_{1,1}+\phi_{2} \\
\phi_{1,3}+\phi_{3}
\end{array}\right]_{2 \times 27}}
\end{aligned}
$$

$$
\begin{aligned}
& {\left[D_{t}\right]=2 b\left[\begin{array}{cc}
k_{1}^{2} c_{66} & 0 \\
0 & \bar{k}_{3}^{2} \bar{c}_{44}
\end{array}\right]} \\
& {\left[D_{b}\right]=\frac{2}{3} b^{3}\left[\begin{array}{ccc}
\hat{c}_{11} & \hat{c}_{13} & 0 \\
\hat{c}_{13} & \hat{c}_{33} & 0 \\
0 & 0 & \hat{c}_{55}
\end{array}\right]}
\end{aligned}
$$

$k_{1}^{2}=\pi^{2}(1+3 R) / 12(1+R)^{2}$

$\bar{k}_{3}^{2}=k_{3}^{2}(1+3 R) /\left(1+\frac{12}{\pi^{2}} k_{3}^{2} R\right)^{2}$

$k_{3}^{2}=\pi^{2}\left\{c_{22}+c_{44}-\left[\left(c_{22}-c_{44}\right)^{2}+4 c_{24}^{2}\right]^{1 / 2}\right\} / 24 \bar{c}_{44}$

$\bar{c}_{p q}=c_{p q}-c_{2 p} c_{q 2} / c_{22}$ 


$$
\hat{c}_{p q}=\bar{c}_{p q}-\bar{c}_{4 p} \bar{c}_{q 4} / \bar{c}_{44}, \quad \hat{c}_{55}=c_{55}-c_{56}^{2} / c_{66}
$$

For the piezoelectric effect, we need to extend the pure mechanical equations of motion to include piezoelectric term [4]. The constitutive equations of piezoelectric media in matrix form are:

$$
\begin{aligned}
& \{T\}=\left[c^{E}\right]\{S\}-[e]\{E\} \\
& \{D\}=[e]^{T}\{S\}-\left[\varepsilon^{S}\right]\{E\}
\end{aligned}
$$

where

$$
\begin{array}{ll}
\mathrm{T} & \text { mechanical stress } \\
\mathrm{S} & \text { mechanical strain } \\
\mathrm{E} & \text { electric field } \\
\mathrm{D} & \text { dielectric displacement } \\
\mathrm{c}^{\mathrm{E}} & \text { mechanical stiffness matrix for constant } \mathrm{E} \\
\mathcal{E}^{S} & \text { permittivity matrix for constant } \mathrm{S} \\
\text { e } & \text { piezoelectric stress constant matrix }
\end{array}
$$

The electric field $\mathrm{E}$ can be expressed by the electrical potential $\mathrm{V}$ :

$$
E=-\nabla V
$$

and the mechanical strain $\mathrm{S}$ to the mechanical displacement $\mathrm{u}$ by:

$$
S=B u
$$

where in the Cartesian coordinates:

$$
B=\left[\begin{array}{cccccc}
\partial / \partial x & 0 & 0 & 0 & \partial / \partial z & \partial / \partial y \\
0 & \partial / \partial y & 0 & \partial / \partial z & 0 & \partial / \partial x \\
0 & 0 & \partial / \partial z & \partial / \partial y & \partial / \partial x & 0
\end{array}\right]^{T}
$$

By Newton's $2^{\text {nd }}$ Law

$$
\nabla T=\rho \partial^{2} u / \partial t^{2}
$$

where $\rho$ is the density of the piezoelectric medium. By Maxwell's equation (no free volume charge in the piezoelectric medium):

$$
\nabla D=0
$$

Now, each node has four degrees of freedom: one displacement, two rotating angle, and one electrical potential. By equations (9)-(13), we can obtain the governing equations for piezoelectric medium. The equation of motion for one element:

$$
\left[\begin{array}{cc}
M^{e} & 0 \\
0 & 0
\end{array}\right]\left\{\begin{array}{l}
\ddot{U}^{e} \\
\ddot{V}^{e}
\end{array}\right\}+\left[\begin{array}{cc}
K^{e} & K_{u V}^{e} \\
K_{u V}^{e}{ }^{T} & K_{V V}^{e}
\end{array}\right]\left\{\begin{array}{l}
U^{e} \\
V^{e}
\end{array}\right\}=\left\{\begin{array}{l}
F^{e} \\
Q^{e}
\end{array}\right\}
$$

where

$$
V=\left\{\begin{array}{lllll}
V_{1} & V_{2} & V_{3} & \cdots & V_{9}
\end{array}\right\}^{T} \quad \text { electrical potential of each }
$$

$$
\begin{aligned}
& K_{u V}^{e}=\int_{A} B_{u}^{T} e^{T} B_{V} d A \\
& B_{u}=B \phi \\
& B_{V}=\left\{\begin{array}{l}
\partial / \partial x \\
\partial / \partial y \\
\partial / \partial z
\end{array}\right\}\left[\begin{array}{lllll}
\varphi_{1} & \varphi_{2} & \varphi_{3} & \cdots & \varphi_{9}
\end{array}\right] \\
& K_{V V}^{e}=\int_{A} B_{V}^{T} \varepsilon^{S} B_{V} d A \\
& F^{e} \quad \text { external force on each node } \\
& Q^{e} \quad \text { electrical charge on each node }
\end{aligned}
$$

in the first term of left hand side, there are zeros in this matrix (no mass for electrical potential), so choosing a suitable method to solve this problem is very important. We do not intend to solve these equations directly, but introduce the "weak coupling" character of quartz to solve the pure mechanical vibration and electrical potential separately.

\section{SOLVING THE EQUATIONS OF MOTION WITH PIEZOELECTRIC EFFECT}

After assembling the whole elements, we can get the equations of motion in matrix form of the quartz plate:

$$
\left[\begin{array}{cc}
M & 0 \\
0 & 0
\end{array}\right]\left\{\begin{array}{l}
\ddot{U} \\
\ddot{V}
\end{array}\right\}+\left[\begin{array}{cc}
K & K_{u V} \\
K_{u V}{ }^{T} & K_{V V}
\end{array}\right]\left\{\begin{array}{l}
U \\
V
\end{array}\right\}=\left\{\begin{array}{l}
F \\
Q
\end{array}\right\}
$$

symbols in (15) without superscript e means for the whole finite element model. In our model, we consider no external force, so $\{F\}=0$; nodes of elements with electrodes (plated): $\{V\}=$ constant ; nodes of elements without electrodes (unplated): $\{V\}=0 ;\{Q\}$ is unknown to be solved.

Based on weak piezoelectric coupling, we solve the mechanical and electrical potential parts separately. First we solve the pure mechanical vibration as an eigenmode problem.

$$
[M]\{\ddot{U}\}+[K]\{U\}=\{0\}
$$

the eigenfunctions

$$
\omega_{f}^{2}[M]\{U\}+[K]\{U\}=\{0\}
$$

where $\omega_{f}$ is the angular frequency of the quartz plate. By solving (17), we can get the eiengmodes (no external force), $U_{1}, U_{2}, U_{1}, \cdots U_{n}$, and the eigenvalues, $\omega_{f 1}, \omega_{f 2}, \omega_{f 3}, \cdots \omega_{f n}$. Finishing the pure mechanical vibration analysis, we can get only natural frequencies and shapes in free vibration, but no response amplitude. Many numerical modes even do not exist in the measured results.

To get the electrical potential, we rearrange the electrical potential to the right hand side of the elastic-piezoelectric equations of motion. These terms become the forcing terms of the pure elastic equations of motion:

$$
\left[K_{u V}\right]\{V(\omega)\}=\left\{F_{e}(\omega)\right\}
$$


in (18), we consider the applied electrical potential $\mathrm{V}$ is sinusoidal $V(\omega)$; the subscript $\mathrm{e}$ in $\mathrm{F}$ means the applied force by electrical potential. Add the forcing and damping term into the equations of motion:

$$
\omega^{2}[M]\left\{U_{p i e}\right\}+j \omega[\eta]\left\{U_{p i e}\right\}+[K]\left\{U_{p i e}\right\}=\left\{F_{e}(\omega)\right\}
$$

the subscript pie means the displacement $U$ with piezoelectric effect. In this paper, we consider the damping is constant. Using the mode superposition method [5] to solve $U_{p i e}$ :

$$
U_{p i e}=\alpha_{i} U_{i}+\alpha_{i+1} U_{i+1}+\alpha_{i+2} U_{i+2}+\cdots+\alpha_{k} U_{k}
$$

where $U_{i}, U_{i+1}, U_{i+2}, \cdots, U_{k}$ are the free vibration eigenmodes we choose to superpose; $\alpha_{i}, \alpha_{i+1}, \alpha_{i+2}, \cdots, \alpha_{k}$ are weights.

We use eigen-expansion to decouple (19) to get decoupled ODEs for the weight of each eigenmode. The elastic displacement field with electrical potential effect, $U_{p i e}$, can then be calculated as the weighting sum of each eigenmode. The electrical charges on the quartz crystal resonator can be obtained from the elastic displacement and electrical potential field at each node point:

$$
\left[\begin{array}{cc}
K_{u V}{ }^{T} & K_{V V}
\end{array}\right]\left\{\begin{array}{c}
U_{p i e} \\
V
\end{array}\right\}=\{Q(\omega)\}
$$

\section{SimUlation RESUlTS}

By this method, we develop a FORTRAN program to calculate the finite element simulation. The case we present in this paper is a $26 \mathrm{MHz}\left(3.5 \times 1.86 \mathrm{~mm}^{2}\right)$ quartz crystal resonator. Its electrode dimension is $1.55 \times 1.2 \mathrm{~mm}^{2}$ and it is bonded on its two short edges. Because of the symmetry in geometric, we can only model the quarter plate to save memory and enhance the efficiency.

\section{A. Convergence Test}

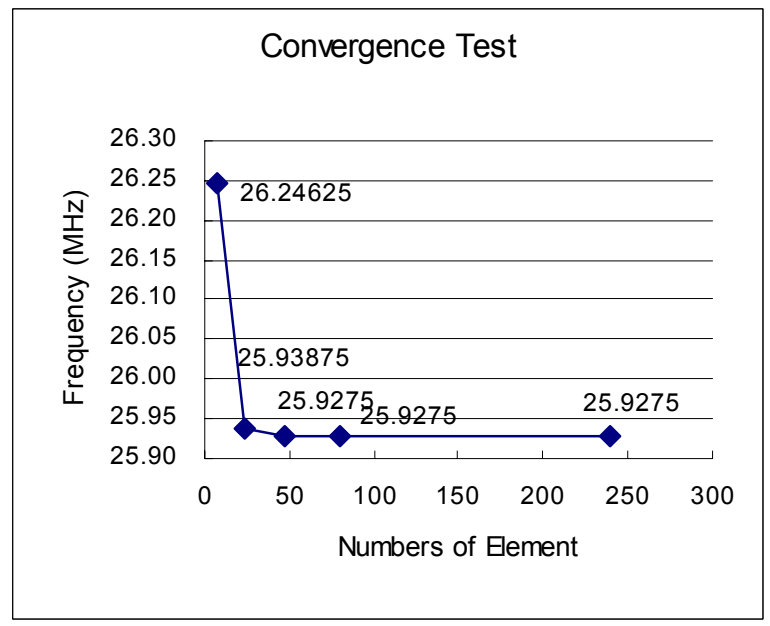

Figure 2. Convergence test for the program in this $26 \mathrm{MHz}$ case
The convergence test shows that as the element number over 50, the major vibration mode (thickness shear) frequency will converge to $25.9275 \mathrm{MHz}$.

\section{B. Comparison Between Simulation and Test Results}

Figure 3 is the test results of the $26 \mathrm{MHz}$ resonator measured by Agilent E5100A. It shows that the major vibration frequency is $25.987 \mathrm{MHz}$, and there are two strong spurious in 26.33817 MHz and 26.745195 MHz

Figure 4 is the simulation results. The major vibration frequency is $25.93875 \mathrm{MHz}$, and there are also two strong spurious. The two spurs are in $26.31375 \mathrm{MHz}$ and 26.83500 $\mathrm{MHz}$ respectively. This simulation results coincide with the test one.

Figure $5 \mathrm{a}$ is the legend of Figures $5 \mathrm{~b}-5 \mathrm{~d}$. It shows the quarter model of the strip quartz plate.

Figures $5 b-5 d$ show the mode shapes of these three resonance frequency. The major vibration in $25.93875 \mathrm{MHz}$ is thickness shear mode; the second, $26.31375 \mathrm{MHz}$, is flexure mode; and the third, $26.83500 \mathrm{MHz}$, is thickness twist mode. The program can obtain the electrical potential response but also the vibration mode shape with piezoelectric effect.

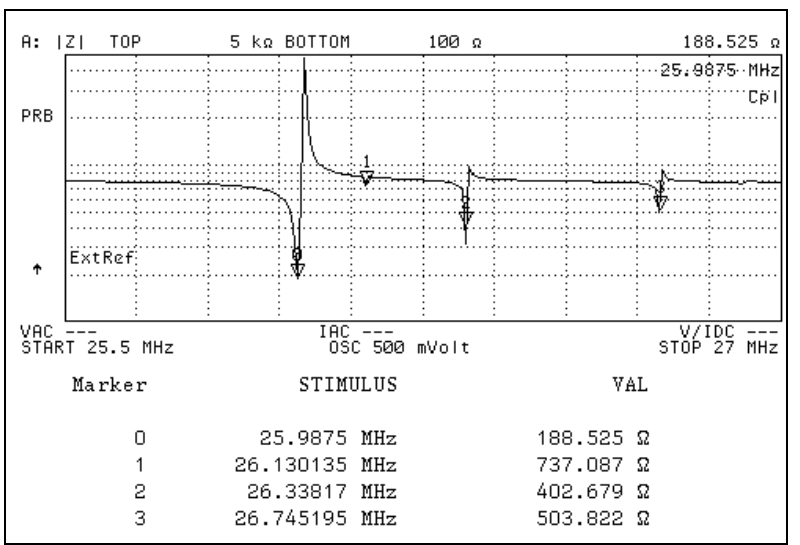

Figure 3 . Test results of the $26 \mathrm{MHz}$ strip quartz resonator

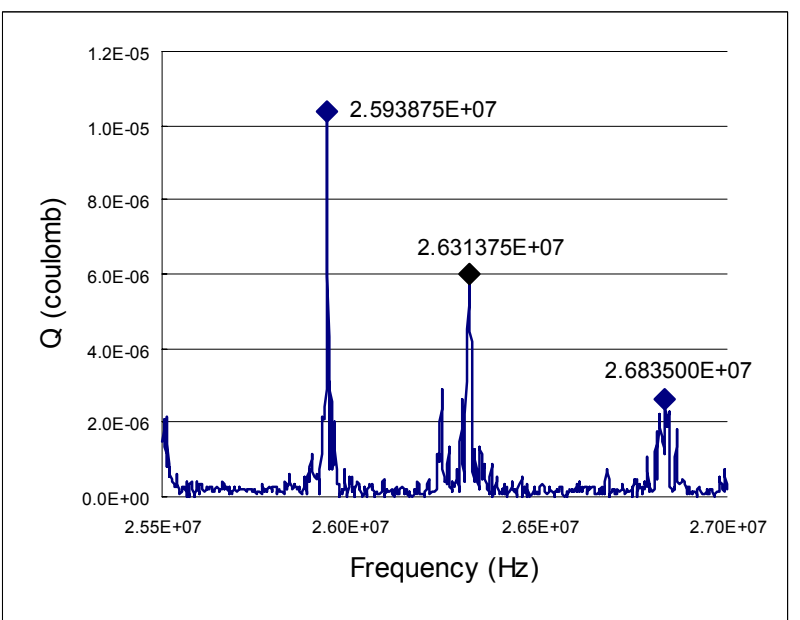

Figure 4. Simulation results of the $26 \mathrm{MHz}$ strip quartz resonator and Frequency Control Joint 50th Anniversary Conference 


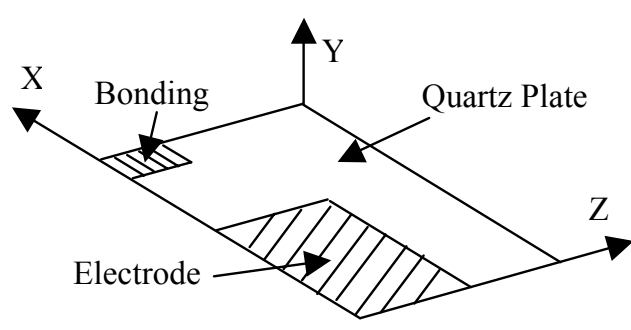

Figure 5a. The legend of the mode shape charts

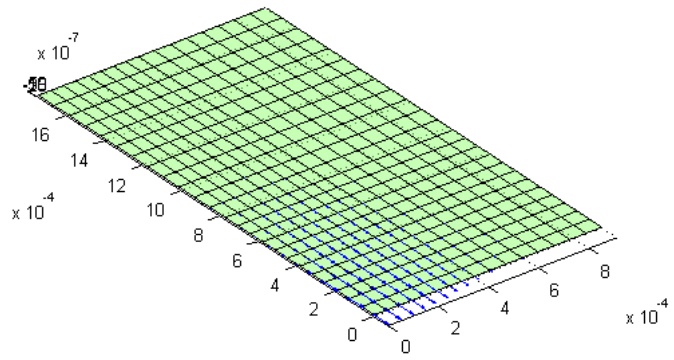

Figure $5 \mathrm{~b}$. The mode shape of the major vibration- thickness shear, 25.93875 MHz. The displacement field is concentrated in the plated area, and the displacements are all in the same $\mathrm{X}$ direction.

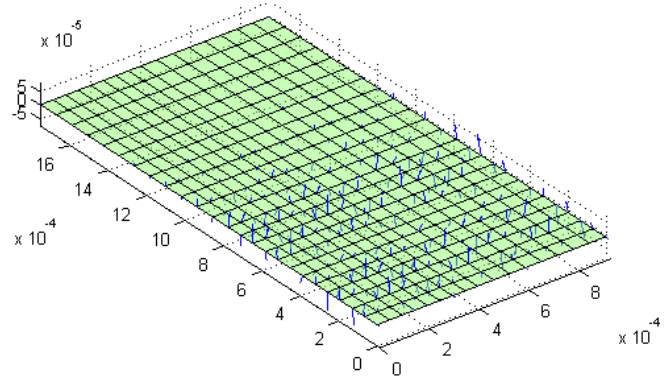

Figure $5 \mathrm{c}$. The mode shape of the second resonance point- flexure, 26.31375 MHz. The displacements are in the out of plane Y direction.

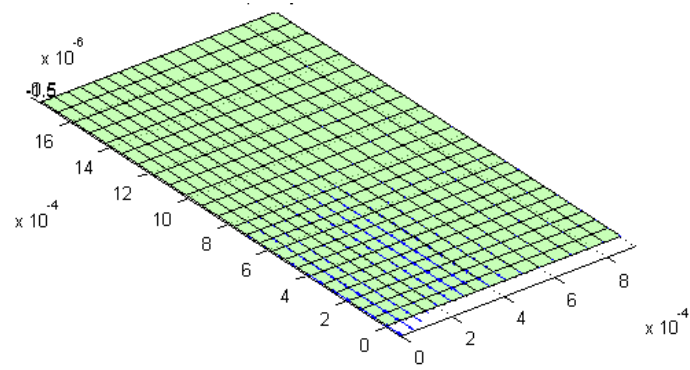

Figure $5 \mathrm{~d}$. The mode shape of the third resonance point- thickness twist, $26.83500 \mathrm{MHz}$. The displacement field is concentrated in the plated area, but the displacements are in different $+\mathrm{X}$ and $-\mathrm{X}$ direction.

\section{CONCLUSION}

Based on Mindlin 2-D elastic-piezoelectric model and weak piezoelectric coupling assumption, we separate the pure mechanical vibration and electrical potential effect to develop an efficient numerical method to analyze the AT-cut quartz resonator. First, we solve the free vibration eigenmodes without piezoelectric effect. Second, rearrange the electrical potential and treat them as forcing terms, and then use the mode superposition method to solve the weak coupling vibration mode shape. By the displacement field, we can get charges on the quartz crystal resonator and the electrical impedance. According to the simulation and test results, this method can analyze AT-cut quartz resonator response with piezoelectric effect and electrode very well. In the future, it can be applied to analyze beveling blanks. It is both precise and efficient enough for design engineers.

\section{REFERENCES}

[1] R.D. Mindlin, "High Frequency Vibrations of Crystal Plates," Quarterly Appl. Math., Vol. 19, pp. 51-61, 1961.

[2] J.N. Reddy, An Introduction to the Finite Element Method, 2nd ed., McGraw-Hill, 1993, pp. 411-416.

[3] P.C.Y. Lee, C. Zee, and C.A. Brebbia, "Thickness-Shear, ThicknessTwist, and Flexural Vibration of Rectangular AT-Cut Quartz Plates with Patch Electrides," 1978 IEEE Tnt. Freq. Control. Symp, pp. 108-119, 1978.

[4] R. Lerch, "Simulation of Piezoelectric Device by Two- and ThreeDimensional Finite Elements," IEEE Trans. UFFC, Vol. 37, No. 2, May 1990.

[5] A.K. Chopra, Dynamics of Structures, Prentice Hall, pp. 434-439 